\title{
On the mechanical behaviour of additively manufactured metamaterials under dynamic conditions
}

\author{
R. Sancho ${ }^{1,2 *}, F$. Galvez ${ }^{1,2}$, C.L. Garrido ${ }^{3}, S$. Perosanz-Amarillo ${ }^{3}$ and D. Barba ${ }^{3}$ \\ ${ }^{1}$ Department of Materials Science, ETSI de Caminos, Canales y Puertos, Universidad Politécnica de \\ Madrid, C/ Profesor Aranguren 3, 28040 Madrid, Spain. \\ ${ }^{2}$ Research Center for Structural Materials (CIME), Universidad Politécnica de Madrid, C/ Profesor \\ Aranguren 3, 28040 Madrid, Spain. \\ ${ }^{3}$ Department of Aerospace Materials and Manufacturing, ETSI de Aeronaútica y del Espacio, \\ Universidad Politécnica de Madrid, Plaza Cardenal Cisneros 3, 28040 Madrid, Spain.
}

\begin{abstract}
High-energy absorption and light-weightiness are two critical properties for impact protection in the aerospace sector. In the past, the use of periodic honeycomb structures or random porous metallic foams were the preferred route to obtain a good specific-energy absorption performance. In recent years, the use of additive manufacturing has increased the design freedom creating a new generation of reticulated and porous materials: the metamaterials or lattice materials. The internal geometries of these lattice structures can be tuned for superior optimal properties, e.g., energyabsorption and density. However, the mechanics of these materials under impact need to be understood with the purpose of mechanical optimisation, and the computational models validated. In this work, we present the experimental compressive behaviour, at room temperature, of two Ti6Al4V lattice structures under static and dynamic conditions. The quasi-static tests were performed by using a universal testing machine while the dynamic tests were conducted at $480 \mathrm{~s}^{-1}$ with a split-Hopkinson bar. In all cases, the deformation process was filmed to analyse the failure. Finally, finiteelement simulations were done, employing the Johnson-Cook model, to describe the response of the alloy. The simulations were able to reflect the failure characteristics of each metamaterial but were not able to describe the macroscopic response due to the differences between the experimental and computational volume fraction.
\end{abstract}

\section{Introduction}

Metamaterials are human-arranged structures with physical properties unseen in nature which are called to revolutionise several strategic sectors like the aerospace, transport, medicine, and energy industries. These structures are usually made up of struts and nodes (reticular or lattice metamaterials), although other complex geometries such as plate-lattices or topological triple minimal surfaces are also part of this material family. The disruptive additive manufacturing of metals has boosted the metamaterial field in the last decade. 
One of the most promising properties of this kind of structures are the light-weightiness and the good specific mechanical properties compared with the bulk material. Nevertheless, other functional properties such as heat exchanging coefficients or biocompatibility can also be improved through the smart design of these porous structures. This means that the door to a new range of multifunctional components has been opened with metamaterials. Some examples of the integration of several functionalities in a component would be the use of metamaterials in light-weight structures integrating heat dissipation and crashworthiness [1], the use of metamaterials in biomedical devices [2,3] or the use of metamaterials in aerospace structures integrating structural, fuel management and impact protection capabilities [4].

However, these lattice structures present several complexities, i.e., components with different characteristic lengths, orientations and microstructures, which lead to different mechanical properties [5]. Understanding the influence of printing parameters and lattice geometry (struts size, orientations, etc.) on the mechanical performance of these materials is an essential requirement to create and design new mechanic metamaterials with optimal properties for the new technological challenges. In this regard, many researchers have studied and linked the static mechanical properties of lattice materials with their geometry and porosity [6 - 10], but much less work has been done in the high-strain rate loading regime $[11,12]$. Therefore, in the present work, the authors study the energy absorption and failure mode of two Ti6Al4V lattice structures under static and dynamic conditions to provide new understanding of the dynamic behaviour of these metamaterials and to rationalise the link between lattice-performance binomial under dynamic conditions. Moreover, an already calibrated Johnson-Cook model was used to simulate all the experimental campaign to gain insights of the effect of design and geometrical parameters on the mechanical performance of the lattice. Having an efficient and reliable computational model is essential for metamaterial optimization.

\section{Materials and experimental methods}

In this study, the investigated specimens consisted of two lattice structures made of Ti6Al4V alloy. This alloy was chosen because of its widely use in aerospace and biomedical sector. The samples were built using the Selective Laser Melting (SLM) technique and specifically, employing a Renishaw AM400 machine with a modulated 200W ytterbium fibre laser. More information about the sample manufacturing, printing parameters and effect of size and orientation on Ti6Al4V can be found in [5].

The lattice structures were additively manufactured with random unit cells. Random unit cells provide metamaterials with a more isotropy behaviour compared with lattices composed of periodic unit cells. An image of the two samples studied in this work is shown in Fig.1, while Table 1 collects their characteristics in terms of solid fractions and dimensions. Discrepancies between the designed and real (after manufacturing) relative density of the lattice structures was identified. This deviation has its origin in the retained powder inside the pores of the lattice during the additive manufacturing process, a common manufacturing issue in small reticular structures.

Table 1. Characteristics of the lattice structures.

\begin{tabular}{|c|c|c|c|}
\hline Lattice & Designed relative density & Real relative density & Dimensions (mm) \\
\hline L & 0.30 & 0.56 & $10 \times 10 \times 16$ \\
\hline N & 0.28 & 0.56 & $10 \times 10 \times 16$ \\
\hline
\end{tabular}




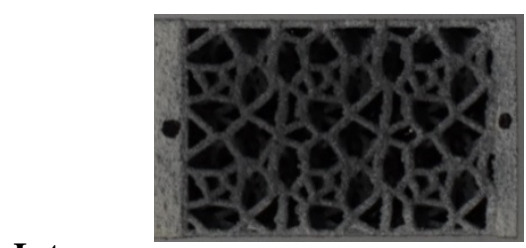

L-type

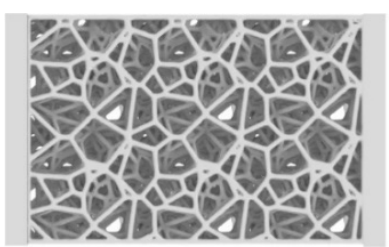

N-type
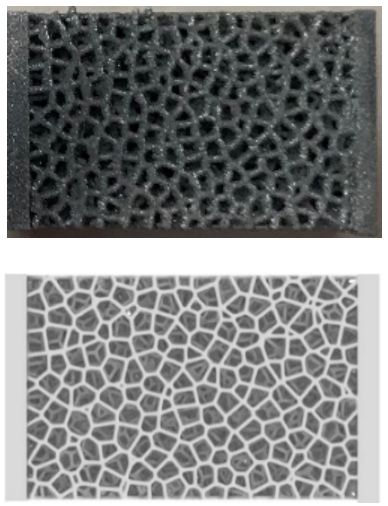

Fig. 1. Lattice structures (real and STL) used in the present study

\subsection{Experimental methods.}

The strain-rate sensitivity on the compressive behaviour of the present lattice materials was characterised through quasi-static and dynamic-loading tests at room temperature. Since the tests reported in this work belong to a preliminary study, only one experiment was conducted for each condition and lattice geometry.

Quasi-static tests were done using a servo-hydraulic MTS universal testing machine equipped with a $100 \mathrm{kN}$ load cell. The samples were loaded at a velocity of $0.014 \mathrm{~mm} / \mathrm{s}$, leading to an engineering strain rate of $0.001 \mathrm{~s}^{-1}$. Strain was monitored using digital image correlation (DIC) tracking the sample. On the other hand, the high strain-rate tests were carried out on a Split Hopkinson Pressure Bar (SHPB) system [13]. In this work, the samples were loaded, approximately, at a velocity of $8000 \mathrm{~mm} / \mathrm{s}$, leading to an engineering strain rate of $480 \mathrm{~s}^{-1}$. The schematic of the SHPB set-up that was used is shown in Fig.2. The system comprises an input and an output bar, both made of maraging C250 steel and a projectile made of another steel, which is responsible for creating the incident compressive stress pulse in the input bar. The specimen was placed between the input and output bar and some lubricant was used to reduce the friction at the bar/specimen interface.

Using the signals from the strain gauges on the bars and applying the theory of 1D-elastic wave propagation [13], the force and the displacement applied to the specimen was calculated with the equations:

$$
\begin{gathered}
F=E_{b} A_{b} \varepsilon_{t} \approx E_{b} A_{b}\left(\varepsilon_{i}+\varepsilon_{r}\right) \\
\Delta l=-2 c_{0} \int_{0}^{t} \varepsilon_{r} d t
\end{gathered}
$$

where $E_{b}$ is the Young's modulus, $A_{b}$ the cross-sectional area and $c_{0}$ the wave velocity of the bars, and $\varepsilon_{i}, \varepsilon_{r}, \varepsilon_{t}$ refers to the incident, reflected and transmitted strain pulses measured on the bars. In the experiments of the present work, a Phantom VE0710 high-speed camera was used to record the failure of the sample during testing at $110000 \mathrm{fps}$ and 256x176 pixels resolution. 


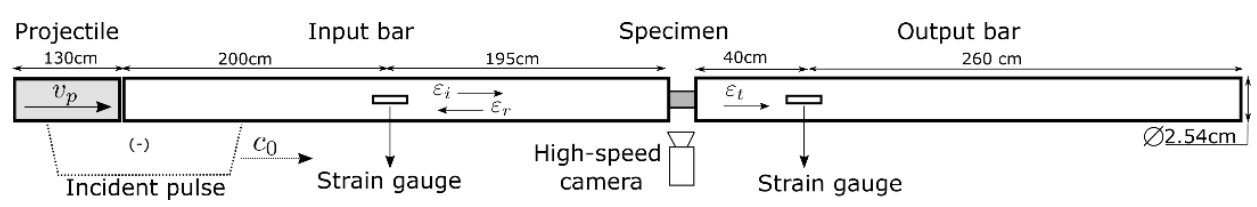

Fig. 2. Schematic of the split Hopkinson Pressure Bar set-up. The projectile is accelerated with compressed air.

\section{Numerical simulations.}

Numerical simulations of the experimental tests were performed by using the commercial finite-element (FE) code ABAQUS with a dynamic-explicit time integration procedure accounting for dynamic effects. To decrease the computational time, the quasi-static simulations were mass-scaled. The FE models included only the lattice beams (not the grip plates of the experimental ones), which were meshed with ten-node tetrahedral elements (C3D10). This type of elements is highly recommended over the linear version (C3D4) to get accurate results when simulating lattice materials subjected to compressive loading [14]. The N-type specimens were modelled with 939,172 elements while the L-type specimens were discretised with 978,306 elements. The boundary conditions were applied on the top and bottom faces of the specimens and were defined to mimic the experimental boundary conditions. In the case of the quasi-static tests, a displacement-based boundary condition was applied to the top face, while a velocity-based boundary condition was chosen for the high strain-rate simulations. Since the displacement (or velocity) of the bottom face in the loading direction was restricted in all simulations, the velocity profile was calculated as the difference between the one measured in the input and output bar ends. The rotations about the axis perpendicular to the loading direction in both, the top and the bottom face, were also restricted. Moreover, unwanted translational movements were avoided by pinning a corner node of the bottom face.

The Johnson-Cook material model $[15,16]$ was selected, due to its extended use in the field of dynamic loading simulations, to define the flow and failure of Ti6Al4V. The material parameters employed in the present work were obtained from [17] and are collected in Table 2. For the shake of simplicity, the element erosion was set to occur when the Johnson-Cook damage initiation criteria was fulfilled (see equation (3)).

$$
D=\int \frac{d \bar{\varepsilon}_{p}}{\varepsilon_{p, f}\left(\sigma^{*}, \dot{\bar{\varepsilon}}_{p}, T\right)}=1
$$

Finally, the possible interactions between the elements during the simulation were taken into account through the general contact option available in Abaqus with the default Normal Behaviour > "Hard" contact properties.

Table 2. Parameters used for the Johnson-Cook model for the Ti6Al4V alloy.

\begin{tabular}{|c|c|c|c|c|c|c|c|}
\hline $\mathbf{A}(\mathbf{M P a})$ & $\mathbf{B}(\mathbf{M P a})$ & $\mathbf{n}$ & $\mathbf{C}$ & $\mathbf{m}$ & $\dot{\boldsymbol{\varepsilon}}_{\mathbf{0}}\left(\mathbf{s}^{\mathbf{- 1}}\right)$ & $\mathbf{T}_{\mathbf{m}} \mathbf{( K )}$ & $\mathbf{T r} \mathbf{( K )}$ \\
\hline 910 & 780 & 0.65 & 0.015 & 0.79 & 0.001 & 1926 & 298 \\
\hline $\mathbf{D}_{\mathbf{1}}$ & $\mathbf{D}_{\mathbf{2}}$ & $\mathbf{D}_{\mathbf{3}}$ & $\mathbf{D}_{\mathbf{4}}$ & $\mathbf{D}_{\mathbf{5}}$ & $\chi$ & $\mathbf{C}_{\mathbf{p}} \mathbf{( J / \mathbf { k g } / \mathbf { K }}$ & $\mathbf{E} \mathbf{( G P a )}$ \\
\hline 0.07 & 0.2 & 0.6 & 0.03 & 4.9 & 0.9 & 520 & 112.6 \\
\hline
\end{tabular}




\section{Results and discussion}

The experimental engineering stress-strain curves of the studied metamaterials are depicted in Fig. 3. It is important to note that the unloading line in the high strain-rate (HSR) curves has to do with the unloading part of the incident pulse and not with load-bearing capacity of the samples. The experimental curves initially increase almost linearly until the first peak. At this point, the bars and nodes of the reticular structure start to fail, leading to the first stress valley. Subsequent stress peaks and valleys occurs during the whole deformation process as a consequence of further beam failures, being these oscillations more prominent under the dynamic regime. A sequence of images that show the failure process of the lattices during dynamic loading are gathered in Fig.4. It is worth noting that the L-type structures fail along two planes perpendicular to the loading direction, first a plane collapses and then the second plane starts failing, while in the N-type structure the failure is concentrated in a single plane that tends to be oriented at $45^{\circ}$. A similar failure sequence was observed in the quasi-static tests.

Regarding the energy-absorption performance, the specimen with small pore-size (N-type sample) absorbs more energy during both loading situations. Moreover, the strain-rate shows a certain negative impact on the energy absorption. The reason of this behaviour is based on the fact that fracture dominates the response of the samples from the very beginning and therefore, the positive strain-rate sensitivity of the inelastic response of the base material (Ti6Al4V alloy) is unnoticed. A negligible strain-rate sensitive on Ti6Al4V plate-lattices was already reported in [12]. The quantitative data of the amount of energy absorbed (per volume) by each lattice under each loading condition, up to a strain equal to $\varepsilon=0.2$, is presented in Table 3.

The numerical results are also presented in Fig. 3 and Fig. 4. There is some similarity regarding the shape of the curves, especially in the first stage of the L-type structure, and the failure sequence during deformation; but there is an important deviation in the stress values that can be explained considering the different relative densities between the experimental and computational specimens (see Table 1.). Although it seems a feasible explanation, we cannot underestimate the need of using a more accurate failure model that takes into account the influence of the Lode angle parameter [12].
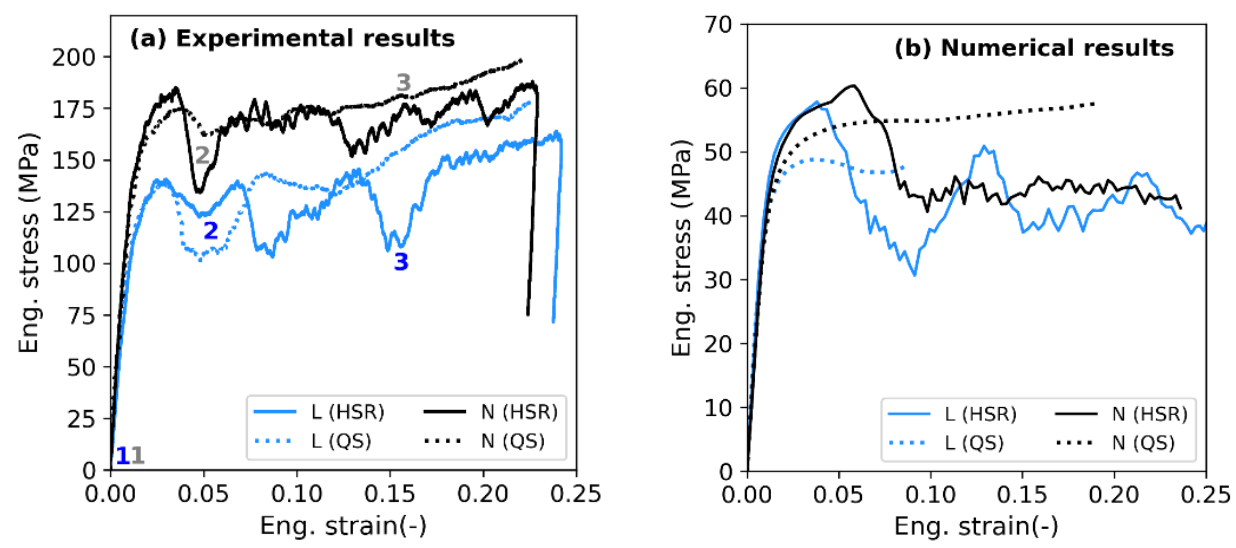

Fig. 3. Experimental and numerical engineering stress-strain curves. 

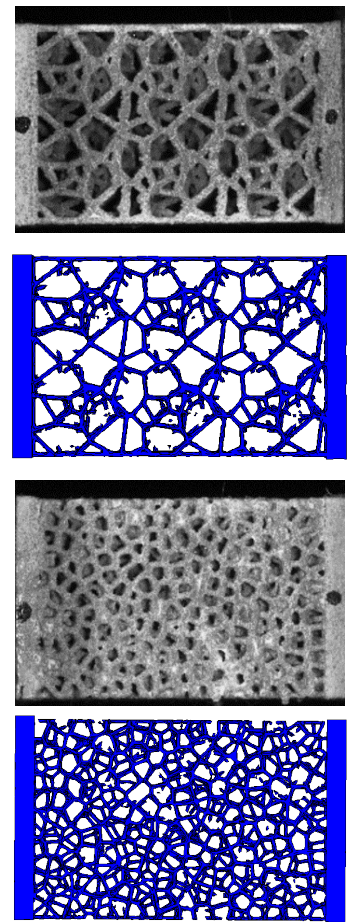
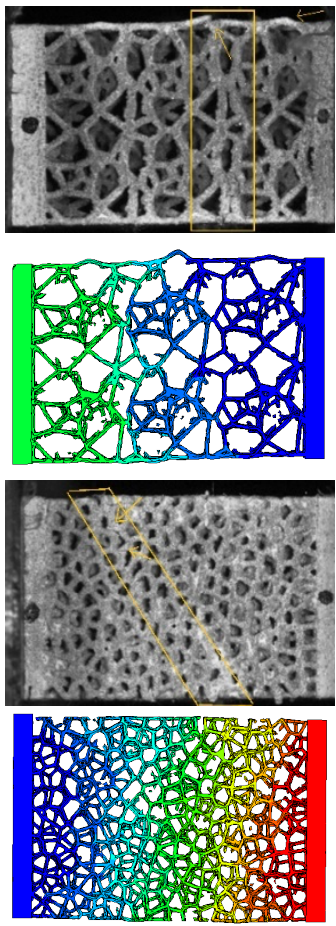
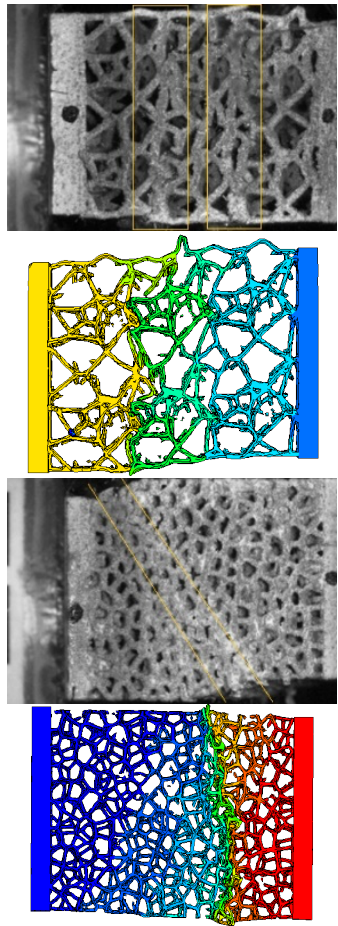

Fig. 4. Experimental and numerical deformation sequence during high strain-rate loading at strain levels of 1) 0 ,2) 0.05 and 3) 0.15 . Colors represent the displacement field in the loading direction.

\begin{tabular}{|c|c|c|c|c|}
\hline \multicolumn{5}{|c|}{ Table 3. Energy absorbed by each type of lattice structure and loading rate. } \\
\hline Lattice & QS $\left(\mathbf{J} / \mathbf{c m}^{3}\right)$ & QS $\left(\mathbf{J} / \mathbf{c m}^{3}\right)$-Specific- & HSR $\left(\mathbf{J} / \mathbf{c m}^{\mathbf{3}}\right)$ & HSR $\left(\mathbf{J} / \mathbf{c m}^{\mathbf{3}}\right)$-Specific- \\
\hline L & 27.01 & 47.86 & 25.18 & 44.96 \\
\hline N & 33.62 & 60.03 & 32.52 & 58.07 \\
\hline
\end{tabular}

\section{Conclusions}

In this work, the quasi-static- and dynamic-loading compressive performance of two Ti6Al4V lattice structures with random unit cells is presented in conjunction with numerical results obtained with a Johnson-Cook material model. The following conclusions can be drawn:

- The lattice structure with small pore-size (type N) absorbs more energy. Its failure occurs along a single $45^{\circ}$ plane, while two failure planes perpendicular to the loading direction appears on L-type lattices.

- High strain-rate has a negative effect on the energy-absorption. Fracture of the truss bars/nodes generates stress-strain curves with peaks and valleys.

- The numerical results cannot be used to validate the suitability of the JohnsonCook model. The general shape of the stress-strain curves and the failure sequence is captured, but the magnitude of the predicted stresses is lower. This difference is grounded on the difference of the relative densities between the experimental and virtual specimens. 
Acknowledgments

The authors gratefully acknowledge the Ministerio de Ciencia, Innovación y Universidades (MCIU), Agencia Estatal de Investigación (AEI) and Fondo Europeo de Desarrollo Regional (FEDER) for providing financial support for this work under grant PGC2018-097116-A-I00.

The authors are also thankful for the support of Arturo Sánchez y Luis Rodríguez during the experimental testing campaign.

\section{References}

1. J.Y. Ho, K.C. Leong, Int. J. Heat Mass Transf., 150, 119262, (2020)

2. E. Alabort, D. Barba, R.C. Reed, Scr. Mater., 164, 110-114, (2019)

3. D. Barba, E. Alabort, R.C. Reed, Acta Biomater., 97, 637-656, (2019).

4. C.G. Ferro, S.Varetti, G.D. Pasquale, P. Maggiore, Mater. Today Commun., 15, 185$189,(2020)$

5. D. Barba, C. Alabort, Y.T. Tang, M.J. Viscasillas, R.C. Reed, E. Alabort, Mater. Des., 186, 108235, (2020)

6. C.Yan, L.Hao, A.Hussein, P.Young, J. Mech Behav Biomed Mater, 51, 61-73, (2015)

7. S. Xu, J. Shen, S. Zhou, X. Huang, Y.M. Xie, Mater. Des., 93, 443-447, (2016).

8. P. Egan, V.C. Gonella, M. Engensperger, S.J. Ferguson, K. Shea, PLoS ONE, 12, 1-20, (2017).

9. X.Y. Zhang, G. Fang, S. Leeflang, A. Zadpoor, J. Zhou, Acta Biomater 84, 437-452, (2018).

10. B. Lozanovski, M. Leary, P. Tran, D. Shidid, M. Qian, P. Choong, M. Brandt, Mater. Des., 171, 107671, (2019).

11. N. Jin, F. Wang, Y. Wang, B. Zhang, H. Cheng, H. Zhang, Mater. Des., 169, 107655, (2019)

12. T. Tancogne-Dejean, X. Li, M. Diamantopoulou, C.C. Roth, D. Mohr, J. Dyn. Behav. Mater., 5, 361-375, (2019)

13. H. Kolsky, Stress Waves in Solids, (Dover, 1963)

14. G. F. de Vera Conrado and B. C. Daniel, Smart Modelling of Additively Manufactured Metamaterials," in Proceedings of the IEEE 10th International Conference Nanomaterials: Applications \& Properties, NAP, 9-13 November 2020, Sumy, Ukraine (2020).

15. G. R. Johnson, W. H. Cook, A constitutive model and data for metals subjected to large strains, high strain rates and high temperatures, in Proceedings of the $7^{\text {th }}$ Int Sympo. Ballistics, 541-547, (1983)

16. G. R. Johnson, W. H. Cook, Eng. Fract. Mech., 21, 31-48, (1985)

17. S. Perosanz-Amarillo, et al. On the effect of the microstructure on the dynamic behaviour of Ti6Al4V, in Proceedings of the $13^{\text {th }}$ International Conference on mechanical and physical behaviour of materials under dynamic loading, 20-24 September 2021, Madrid, Spain (2021) 